\title{
A new multivariate CUSUM chart using principal components with a revision of Crosier's chart
}

\author{
Jiaqi Chen ${ }^{1}$, Hualong Yang $^{2}$ and Jianfeng Yao $^{2}$ \\ ${ }^{1}$ Department of Mathematics, Harbin Institute of Technology \\ ${ }^{2}$ Department of Statistics and Actuarial Science, University of Hong Kong
}

\begin{abstract}
In this paper, we introduce a new multivariate cumulative sum chart where the target shift mean is assumed to be a weighted sum of principal directions of the population covariance matrix. This chart provides an attractive performance in term of average run length (ARL) for large-dimensional data and it also compares favourably to existing multivariate charts including Crosier's benchmark chart with updated values of the upper control limit and the associated ARL function. In addition, Monte Carlo simulations are conducted to assess the accuracy of the well known Siegmund's approximation of the average ARL function when observations are normal distributed. As a byproduct of the paper, we provide updated values of upper control limits and the associated ARL function for Crosier's multivariate CUSUM chart.
\end{abstract}

KEY WORDS: Average run length; Multivariate CUSUM; Multivariate statistical process control, Crosier's multivariate CUSUM chart. 


\section{Introduction}

Cumulative sum (CUSUM) procedures are traditional sequential procedures for detecting a mean shift in a process. Contributions to its theory have been continuously developed since their original introduction by Page (1954). Recent interests include their extension to control charts that monitor multivariate observations leading to multivariate CUSUM (MCUSUM for short) procedures, see Runger and Testik (2004) and the review paper of Bersimis et al. (2007, Section 3).

More precisely, Healy (1987) developed a procedure using the theory of sequential ratio tests (SRT, actually already mentioned in Page's original paper). This procedure assumes that the direction of the shift is explicitly known. Crosier (1988) introduced a special MCUSUM procedure where a clever definition was given to the positive part of a vector. This procedure, hereafter referred as $\mathrm{MCO}$, has the advantage that the average run lengths $(A R L)$ depend only on the noncentral parameter (instead of on- and off-target means and the population covariance matrix). This procedure was proved to outperform both the so-called COT procedure, also introduced in Crosier (1988), where sums of Hotelling's $T$ (not $T^{2}$ ) are accumulated and the procedure in Woodall and Ncube (1985) which combines several univariate CUSUM charts. Pignatiello and Runger (1990) introduced two other MCUSUM procedures (MC1 and MC2) which are indeed very close, both in spirit and in performance, to the MCO and COT procedures, respectively. Lastly, in a recent paper Golosnoy et al. (2009), several enhanced versions of the MC1 procedure have been introduced.

In this paper, we first re-examine the problem of detection of a mean shift of a multivariate normal distribution and the MCUSUM procedures discussed above. Then, we propose a new MCUSUM procedure based on the SRT scheme as in Healy (1987) but without assuming a rigid direction for the shift in the mean. Indeed, we do not assume that such direction is available; rather, we will assume that possible direction for the shift is distributed as a weighted sum of 
principal component directions. This new procedure is shown to outperform Crosier's MCUSUM procedure. It also compares favourably to the improved versions of the MC1 procedure given in Golosnoy et al. (2009).

\section{Revision of Crosier's MCUSUM procedure}

Crosier's multivariate CUSUM chart has been for long time a benchmark in multivariate process control. Recall first the $n$-th step of a classic univariate CUSUM chart: the accumulated sum is

$$
S_{n}=\max \left\{0, S_{n-1}+\left(X_{n}-\mu_{0}\right)-k \sigma\right\},
$$

where $X_{n}$ is the observation following an univariate normal distribution, $\mu_{0}$ the on-target (in control) mean of the process, $\sigma$ the standard deviation of the process and $k$ is a positive parameter specific to the CUSUM chart. For multivariate observations $\left(\mathbf{x}_{\mathbf{n}}\right)$ of dimension $p$ with covariance matrix $\Sigma$ and on-target mean $\mu_{0}$, a similar formula $\mathbf{s}_{\mathbf{n}}=\max \left\{0, \mathbf{s}_{\mathbf{n}-\mathbf{1}}+\left(\mathbf{x}_{\mathbf{n}}-\mu_{\mathbf{0}}\right)-\mathbf{k}\right\}$ taking the maximum of two vectors would be ill-defined. The solution provided in Crosier (1988) is the following: set $\mathbf{s}_{\mathbf{0}}=\mathbf{0}$ and for $n \geq 1$, first compute the length

$$
C_{n}=\left[\left(\mathbf{s}_{\mathbf{n}-\mathbf{1}}+\mathbf{x}_{\mathbf{n}}-\mu_{\mathbf{0}}\right)^{T} \Sigma^{-1}\left(\mathbf{s}_{\mathbf{n}-\mathbf{1}}+\mathbf{x}_{\mathbf{n}}-\mu_{\mathbf{0}}\right)\right]^{\frac{1}{2}} .
$$

Then, use the rule

$$
\mathbf{S}_{\mathbf{n}}= \begin{cases}\mathbf{0}, & \text { If } C_{n} \leqslant k, \\ \left(\mathbf{s}_{\mathbf{n}-\mathbf{1}}+\mathbf{x}_{\mathbf{n}}-\mu_{\mathbf{0}}\right)\left(1-\frac{k}{C_{n}}\right), & \text { If } C_{n}>k .\end{cases}
$$

Here $k$ is a scalar parameter playing a similar role as in the univariate CUSUM procedure. Indeed, by mimicking the univariate case, this constant $k$ is always fixed to be $k=\frac{1}{2}$. This chart will alarm 
when the length of $\mathbf{s}_{\mathbf{n}}$ exceeds a control limit $H$ i.e. $L_{n}=\left(\mathbf{s}_{\mathbf{n}}{ }^{T} \Sigma^{-1} \mathbf{S}_{\mathbf{n}}\right)^{\frac{1}{2}}>H$. It has also been proved that the distribution of the test statistic $L_{n}$ depends only on the value of the non-centrality parameter $d^{2}=\left(\mu-\mu_{\mathbf{0}}\right)^{T} \Sigma^{-1}\left(\mu-\mu_{\mathbf{0}}\right)$ where $\mu$ is the off-target mean of the process. It follows that the function of the average run length $(A R L)$ of this control chart also depends on $d$ only.

Note that the multivariate CUSUM MC1 in Pignatiello and Runger (1990) is very close to this benchmark procedure. Next, this MC1 chart has been modified further in Golosnoy et al. (2009) where some enhancements have been added. We will present a comparison to these charts in next section.

As Crosier's paper was published nearly 25 years ago, we have found interesting to check whether the original values given by Crosier are still accurate enough given that nowadays we have much more powerful computing facilities. First, we compute by simulations the values of the upper control limit (UCL) $H$. Recall that $H$ is fixed so that $A R L_{0}$, the $A R L$ when the process is under control, equals a given value. Simulations are done for $A R L_{0}=200$ and $A R L_{0}=500$ using 10000 independent replications. These values are given in Table 1 . When $A R L_{0}=200$, the newly revised values are very close to the original values given by Crosier with an exception for the dimension $p=5$. However, when $A R L_{0}=500$, most of revised values are significantly different from the original ones. As mentioned earlier, we believe that such discrepancy is due to differences in computing accuracy of the software Crosier and we used respectively.

Table 1: Values of the UCL $H$ for different dimensions $p$ and $A R L_{0}=200$ or 500.

\begin{tabular}{llcccc} 
& $p$ & 2 & 5 & 10 & 20 \\
\hline \hline$A R L_{0}=200$ & original $H$ & 5.50 & 9.46 & 14.90 & 24.70 \\
& revised $H$ & 5.49 & 9.38 & 14.92 & 24.70 \\
\hline$A R L_{0}=500$ & original $H$ & 6.65 & 10.90 & 17.20 & 28.00 \\
& revised $H$ & 6.56 & 10.90 & 17.09 & 28.11 \\
\hline
\end{tabular}

Secondly, using these revised values of $H$, we have also determined the empirical $A R L$ functions for different dimensions. Note that in Crosier's original paper, these functions are plotted 
only and their explicit values are unknown. These values are now available from this paper, they are believed to be more accurate with modern computing facilities. As for the off-target scenario, we have considered two scenarios for the off-target mean $\mu$ :

1. $\mu=(d, 0, \ldots, 0), \Sigma=\mathbf{I}$.

2. $\mu=\left(\frac{d}{\sqrt{p}}, \frac{d}{\sqrt{p}}, \ldots, \frac{d}{\sqrt{p}}\right), \Sigma=\mathbf{I}$.

Both situations share a same value of non-centrality parameter $d$. The revised values are given in Table 2 for both values of $A R L_{0}=200$ and $A R L_{0}=500$. As predicted by the theory, the $A R L$ function is the same under the two scenarios, and its values quickly decrease as the off-target distance $d$ grows from 0 (in-control) to the value of 4 .

Table 2: Empirical $A R L$ values for two off-target means when $A R L_{0}=200$ (upper block) and $A R L_{0}=200$ (lower block).

\begin{tabular}{|c|c|c|c|c|c|c|c|c|}
\hline \multirow[b]{2}{*}{$d$} & \multicolumn{2}{|c|}{$p=2$} & \multicolumn{2}{|c|}{$p=5$} & \multicolumn{2}{|c|}{$p=10$} & \multicolumn{2}{|c|}{$p=20$} \\
\hline & 1 & 2 & 1 & 2 & 1 & 2 & 1 & 2 \\
\hline 0 & 200.855 & 197.886 & 199.863 & 202.578 & 199.431 & 201.147 & 200.673 & 198.615 \\
\hline 0.5 & 29.539 & 29.776 & 34.937 & 35.047 & 42.647 & 43.108 & 56.327 & 55.949 \\
\hline 1 & 9.865 & 9.840 & 13.527 & 13.587 & 18.662 & 18.697 & 27.259 & 27.120 \\
\hline 1.5 & 5.781 & 5.777 & 8.426 & 8.364 & 11.945 & 11.963 & 17.989 & 17.995 \\
\hline 2 & 4.112 & 4.134 & 6.098 & 6.133 & 8.825 & 8.851 & 13.458 & 13.487 \\
\hline 2.5 & 3.241 & 3.234 & 4.828 & 4.835 & 7.038 & 7.016 & 10.790 & 10.798 \\
\hline 3 & 2.691 & 2.687 & 4.003 & 4.022 & 5.870 & 5.863 & 9.042 & 9.042 \\
\hline 3.5 & 2.325 & 2.326 & 3.483 & 3.466 & 5.052 & 5.046 & 7.787 & 7.796 \\
\hline 4 & 2.097 & 2.101 & 3.075 & 3.079 & 4.455 & 4.446 & 6.682 & 6.856 \\
\hline 0 & 498.504 & 496.057 & 500.075 & 504.420 & 500.700 & 494.300 & 497.197 & 499.702 \\
\hline 0.5 & 39.471 & 40.023 & 46.970 & 46.847 & 56.231 & 56.263 & 73.384 & 73.440 \\
\hline 1 & 11.937 & 11.743 & 16.262 & 16.303 & 22.356 & 22.346 & 32.646 & 32.710 \\
\hline 1.5 & 6.866 & 6.807 & 9.828 & 9.860 & 13.945 & 13.922 & 21.082 & 21.131 \\
\hline 2 & 4.835 & 4.803 & 7.059 & 7.112 & 10.193 & 10.231 & 15.631 & 15.645 \\
\hline 2.5 & 3.766 & 3.769 & 5.564 & 5.579 & 8.072 & 8.065 & 12.453 & 12.452 \\
\hline 3 & 3.127 & 3.125 & 4.652 & 4.634 & 6.708 & 6.710 & 10.363 & 10.369 \\
\hline 3.5 & 2.685 & 2.677 & 3.983 & 3.973 & 5.771 & 5.765 & 8.908 & 8.921 \\
\hline 4 & 2.347 & 2.350 & 3.480 & 3.494 & 5.069 & 5.065 & 7.819 & 7.825 \\
\hline
\end{tabular}




\section{A new MCUSUM chart based on principal components}

In this section we introduce a new MCUSUM procedure inspired by a combination of advantages from the methodology of sequential ratio tests and the popular method of principal components analysis. Let $\left(\mathbf{x}_{\mathbf{k}}\right)$ be a sequence of $p$ dimensional and independent observation vectors following a normal distribution with known covariance matrix $\Sigma$, and on-target mean vector $\mu_{0}$ and offtarget mean vector $\mu_{1}$. To start with and similarly to Healy (1987), consider the sequence of log-likelihood ratios, i.e. ratios of off-target likelihoods over on-target likelihoods

$$
\begin{aligned}
s_{n} & =\ln \frac{f_{\mu_{1}}\left(\mathbf{x}_{\mathbf{n}}\right)}{f_{\mu_{0}}\left(\mathbf{x}_{\mathbf{n}}\right)} \\
& =\left(\mu_{1}-\mu_{0}\right)^{T} \Sigma^{-1}\left(\mathbf{x}_{\mathbf{n}}-\frac{\mu_{1}+\mu_{0}}{2}\right) \\
& =\left(\mu_{1}-\mu_{\mathbf{0}}\right)^{T} \Sigma^{-1}\left(\mathbf{x}_{\mathbf{n}}-\mu_{\mathbf{0}}\right)-\frac{1}{2} d^{2},
\end{aligned}
$$

where $f_{\mu}(\mathbf{x})$ is the density function of $\mathbf{x}$ with mean $\mu$ and

$$
d^{2}=\left(\mu_{1}-\mu_{0}\right)^{T} \Sigma^{-1}\left(\mu_{1}-\mu_{0}\right)
$$

is the Mahahanobis distance between the on-target and off-target distributions (non-centrality parameter).

In Healy's procedure, the direction of shift in the mean $\mu_{1}-\mu_{\mathbf{0}}$ is assumed known explicitly so that by letting $\mathbf{a}=\Sigma^{-1}\left(\mu_{1}-\mu_{\mathbf{0}}\right)$, a computable quantity, the above likelihood ratios are simply

$$
s_{n}=\mathbf{a}^{T} \mathbf{x}_{\mathbf{n}}-\frac{1}{2} d^{2}
$$

Therefore once $d$ is given to a pre-specified value (say 1), one can determine the remaining parameter, namely the UCL $H$ according to an assigned value of $A R L_{0}(A R L$ under the on-target condition). 
It is well-known that in real statistical process control, it is extremely rare that such a direction in a future mean shift is known explicitly in advance. For this reason and despite a real application described in Healy's paper, it seems that his approach has not been much followed by other practitioners and researchers. However, sequential ratio tests have been proved to be an efficient procedure in other statistical problems; they are even optimal when data have a normal distribution, see Wald (2004) and Siegmund (1985). The MCUSUM procedure introduced below is aimed at developing a ratio tests based procedure while relaxing the restrictive direction of shift adopted in Healy (1987).

We thus assume that no prior information on directions of future shifts of the process mean is available. Our procedure will rely heavily on the spectral structure of the population covariance matrix $\Sigma$. To start with, assume for a moment that $\Sigma$ is diagonal,

$$
\Sigma=\left(\begin{array}{cccc}
\sigma_{1}^{2} & 0 & \cdots & 0 \\
0 & \sigma_{2}^{2} & \cdots & 0 \\
0 & 0 & \ddots & 0 \\
0 & \cdots & 0 & \sigma_{p}^{2}
\end{array}\right)
$$

It is then natural to consider a shift of mean of the form

$$
\mu_{1}-\mu_{\mathbf{0}}=\alpha\left(\sigma_{1}, \sigma_{2}, \cdots, \sigma_{n}\right)^{T}
$$

where $\alpha$ is a positive parameter controlling the overall scale. In other words, we focus on directions of shift where each coordinate will shift proportionally to their standard deviation in absence of any prior information.

How we can then adapt this idea to a general covariance matrix? Let be the spectral decomposition of $\Sigma$

$$
\Sigma=\sigma_{1}^{2} \mathbf{u}_{1} \mathbf{u}_{1}^{\mathbf{T}}+\cdots+\sigma_{\mathbf{p}}^{2} \mathbf{u}_{\mathbf{p}} \mathbf{u}_{\mathbf{p}}^{\mathbf{T}}
$$


where $\left\{\sigma_{i}^{2}\right\}$ and $\left\{\mathbf{u}_{\mathbf{i}}\right\}$ are the eigenvalues and the eigenvectors of $\Sigma$, respectively. We will then consider directions of shift of the following form:

$$
\mu_{1}-\mu_{\mathbf{0}}=\alpha\left(\sigma_{1} \mathbf{u}_{1}+\sigma_{2} \mathbf{u}_{2}+\cdots+\sigma_{\mathbf{p}} \mathbf{u}_{\mathbf{p}}\right),
$$

with some scale parameter $\alpha$. The role of this scale parameter will be described in details below. Note already that directions in (4) reduce to those of (2) for a diagonal covariance matrix $\Sigma$. In this sense, (4) is a natural extension of the intuitive idea in (2).

Therefore, for our MCUSUM procedure, we have

$$
d^{2}=\left(\mu_{1}-\mu_{\mathbf{0}}\right)^{T} \Sigma^{-1}\left(\mu_{1}-\mu_{\mathbf{0}}\right)=\alpha^{2} p
$$

By (1), the likelihood ratios have the form

$$
\begin{aligned}
s_{n} & =\left(\mu_{1}-\mu_{0}\right)^{T} \Sigma^{-1}\left(\mathbf{x}_{\mathbf{n}}-\mu_{0}\right)-\frac{1}{2} \alpha^{2} p \\
& =\alpha\left(\frac{1}{\sigma_{1}} u_{1}+\frac{1}{\sigma_{2}} u_{2}+\ldots+\frac{1}{\sigma_{p}} u_{p}\right)^{T}\left(\mathbf{x}_{\mathbf{n}}-\mu_{0}\right)-\frac{1}{2} \alpha^{2} p \\
& =\alpha\left(\frac{1}{\sigma_{1}} c_{n}^{1}+\frac{1}{\sigma_{2}} c_{n}^{2}+\ldots \frac{1}{\sigma_{p}} c_{n}^{p}\right)-\frac{1}{2} \alpha^{2} p \quad\left(k=\frac{1}{2} \alpha^{2} p\right),
\end{aligned}
$$

where the $\left(c_{n}^{j}\right)$ are the coordinates of the new observation $\mathbf{x}_{\mathbf{n}}$ on the principal directions $\left(\mathbf{u}_{\mathbf{j}}\right)$ :

$$
c_{n}^{j}=\mathbf{u}_{\mathbf{j}}^{T}\left(\mathbf{x}_{\mathbf{n}}-\mu_{\mathbf{0}}\right), \quad j=1, \ldots, p .
$$

The relationship between our procedure and the principal component analysis based on the spectral structure of $\Sigma$ is thus visible. 
Finally, our MCUSUM has the form

$$
\begin{aligned}
S_{0} & =0, \\
S_{n} & =\max \left\{S_{k-1}+s_{n}, 0\right\} \\
& =\max \left\{S_{k-1}+\alpha\left(\frac{1}{\sigma_{1}} c_{n}^{1}+\frac{1}{\sigma_{2}} c_{n}^{2}+\ldots \frac{1}{\sigma_{p}} c_{n}^{p}\right)-\frac{1}{2} \alpha^{2} p, 0\right\} .
\end{aligned}
$$

We thus accumulate the sum $S_{n}$ for sample vectors $\mathbf{x}_{\mathbf{n}}$ 's until it exceeds a control limit $H$ (i.e. when $S_{n}>H$ ) where an alarm is then issued. As usual, the UCL $H$ will be determined according to some pre-specified value $A R L_{0}$ of on-target $A R L$ (for example 200).

\section{Determination of the scale parameter $\alpha$}

From Equation (5), it is clear that the parameter $\alpha^{2}$ will control the scale of the mean shift at which the designed chart is aimed. Here we specify two standard choices of this parameter by following common practice employed in existing MCUSUM procedures.

\section{a). Chart for overall unit shifts of the mean vector}

We first consider the scale $\alpha=\frac{1}{\sqrt{p}}$. In this case, $d^{2}=1$ for the non-centrality parameter. In a sense, whatever the dimension $p$ is, the chart is aimed at detecting an overall unit shift of the mean. This setting is the one used in all the existing literature, such as Crosier (1988); Pignatiello and Runger (1990); Golosnoy et al. (2009).

In this case, our MCUSUM procedure has the form

$$
\begin{aligned}
S_{0} & =0, \\
S_{n} & =\max \left\{S_{n-1}+s_{n}, 0\right\} \\
& =\max \left\{S_{n-1}+\frac{1}{\sqrt{p}}\left(\frac{1}{\sigma_{1}} c_{n}^{1}+\frac{1}{\sigma_{2}} c_{n}^{2}+\ldots \frac{1}{\sigma_{p}} c_{n}^{p}\right)-\frac{1}{2}, 0\right\} .
\end{aligned}
$$


This chart will have a better performance when possible shifts of the mean are around the unit value 1 , e.g. $d=0,0.5,1.5, \ldots, n$ for some small integer $n$. Since these values are independent from the dimension $p$, target shifts of the mean are those parallel to one or few principal directions $\mathbf{u}_{j}$.

\section{b). Chart for unit shifts of the mean vector in all principal directions}

Next we develop a chart where the target shifts can happen simultaneously in all the principal directions $\left\{\mathbf{u}_{j}\right\}$. Thus we consider a situation where $\alpha=1$ and the non-centrality parameter becomes $d^{2}=p$. Here the target shifts as well as the resulting MCUSUM procedure will depend on the dimension $p$. A possible scenario for this scheme is that shifts happen in all principal directions $\left\{\mathbf{u}_{j}\right\}$ at scales which are proportional to their associated variances (or inertia), i.e. $\mu_{\mathbf{1}}-\mu_{\mathbf{0}}=\sigma_{1} \mathbf{u}_{1}+\cdots+\sigma_{p} \mathbf{u}_{p}$.

In this case, our MCUSUM procedure has the form

$$
\begin{aligned}
S_{0} & =0, \\
S_{n} & =\max \left\{S_{n-1}+s_{n}, 0\right\} \\
& =\max \left\{S_{n-1}+\frac{1}{\sigma_{1}} c_{n}^{1}+\frac{1}{\sigma_{2}} c_{n}^{2}+\ldots \frac{1}{\sigma_{p}} c_{n}^{p}-\frac{p}{2}, 0\right\} .
\end{aligned}
$$

This chart will have a best performance when possible shifts of the mean are around the dimension $p$, e.g. $d=0,0.5 p, 1.5 p, \ldots, m p$ for some small integer $m$.

\section{Analytic computation of the ARL function}

In order to compare the performance of the new CUSUM charts to existing MCUSUM charts, we will focus on the chart given in (9) because this chart has an order of magnitude for target shifts of the mean identical to the one used in the references. This chart will be hereafter referred 
as MCn chart. Note however that the analytic development below for this chart can be carried out in a similar way for the other chart given in (10).

As quantities we are accumulating have a normal distribution, one analysis of the $A R L$ function will be based on the approximation formula established in Siegmund (1985). These approximation are helpful since they avoid the use of intensive Monte-Carlo simulation which is time-consuming. Siegmund's formula for the $A R L$ function from normal sequential ratio tests are [see formula (5.5.9)-(5.5.10) in Basseville et al. (1993)]:

$$
\begin{aligned}
& \operatorname{ARL}_{\left(\mu^{\prime}=0\right)}=\left(\frac{H}{\sigma^{\prime}}+1.166\right)^{2} \\
& A R L_{\left(\mu^{\prime} \neq 0\right)}=\frac{\sigma^{\prime 2}}{2 \mu^{\prime 2}}\left[e^{-2\left(\frac{\mu^{\prime} H}{\sigma^{\prime 2}}+1.166 \cdot \frac{\mu^{\prime}}{\sigma^{\prime}}\right)}+2\left(\frac{\mu^{\prime} H}{\sigma^{\prime 2}}+1.166 \cdot \frac{\mu^{\prime}}{\sigma^{\prime}}\right)-1\right]
\end{aligned}
$$

where $\mu^{\prime}=\mathrm{E}\left(s_{n}\right)$ and $\sigma^{\prime 2}=\operatorname{Var}\left(s_{n}\right)$. For our MCUSUM procedure, the likelihood ratios have the form

$$
s_{n}=\frac{1}{\sqrt{p}}\left(\frac{1}{\sigma_{1}} c_{n}^{1}+\frac{1}{\sigma_{2}} c_{n}^{2}+\ldots \frac{1}{\sigma_{p}} c_{n}^{p}\right)-\frac{1}{2},
$$

and the associated $A R L$ formula are

$$
\begin{aligned}
& A R L_{\left(d=\frac{1}{2}\right)}=(H+1.166)^{2}, \\
& A R L_{\left(d \neq \frac{1}{2}\right)}=\frac{1}{2(d-0.5)^{2}}\{\exp [-(H+1.166) \cdot(2 d-1)]-1+(H+1.166) \cdot(2 d-1)\} .
\end{aligned}
$$

The derivation of these formula is given in Appendix A. Afterwards, we compute the $H$ making sure that $A R L_{0}$ equals the given value (200 or 500) and then get $A R L$ s for different distance. These values are given in Table 3 . 
Table 3: Siegmund's $A R L$ approximation when $A R L_{0}=200$ or $A R L_{0}=500$.

\begin{tabular}{ccccccccc} 
& \multicolumn{8}{c}{$A R L_{0}=200$} \\
\cline { 2 - 9 }$d$ & 0 & 0.5 & 1 & 1.5 & 2 & 2.5 & 3 & 3.5 \\
$\operatorname{ARL}(d)$ & 200.01 & 21.72 & 7.34 & 4.16 & 2.88 & 2.21 & 1.78 & 1.50 \\
\hline \hline & \multicolumn{8}{c}{$A R L_{0}=500$} \\
\cline { 2 - 9 }$d$ & 0 & 0.5 & 1 & 1.5 & 2 & 2.5 & 3 & 3.5 \\
$A R L(d)$ & 498.96 & 30.75 & 9.10 & 5.05 & 3.47 & 2.65 & 2.14 & 1.79 \\
\hline \hline
\end{tabular}

Finally, we have conducted an extensive simulation experiment to assess the accuracy of Siegmund's approximations (14)-(15). The results are reported in Appendix B.

\section{Comparison between the new MCUSUM chart and previ- ous methods}

First, we compare the new MCUSUM chart MCn to Crosier's MC0 chart. The empirical ARLs and their standard errors (in bracket) are reported in Table 4 for both values of $A R L_{0}=200$ and $A R L_{0}=500$. The comparison has been made for those shifts of the mean and dimensions $p$ reported by Crosier (1988): shifts corresponding to distances $d=0,0.5,1,1.5,2.0,2.5,3$ and dimensions $p=2,3,5,10,20$, respectively. 
Table 4: $A R L$ comparison between the new chart ( $\mathrm{MCn})$ and Crosier's chart $(\mathrm{MC} 0)$ when $A R L_{0}=$ 200 (upper blocks) and $A R L_{0}=500$ (lower blocks) . Numbers in brackets are corresponding standard errors.

\begin{tabular}{|c|c|c|c|c|}
\hline$d$ & $\mathrm{MCO}$ & $\mathrm{MCn}$ & $\mathrm{MCO}$ & $\mathrm{MCn}$ \\
\hline & \multicolumn{2}{|c|}{$p=2$} & \multicolumn{2}{|c|}{$p=5$} \\
\hline 0 & 197.91(193.83) & 198.07(196.10) & $202.39(185.31)$ & $\mathbf{1 9 9 . 1 4}(196.84)$ \\
\hline 0.5 & $29.96(22.37)$ & $21.67(17.65)$ & $34.74(21.62)$ & $\mathbf{2 1 . 7 3}(17.46)$ \\
\hline 1 & $9.82(4.77)$ & $\mathbf{7 . 3 1}(4.18)$ & $13.54(5.10)$ & $\mathbf{7 . 3 3}(4.24)$ \\
\hline 1.5 & $5.80(2.09)$ & 4.27(1.90) & $8.40(2.41)$ & $\mathbf{4 . 2 4}(1.87)$ \\
\hline 2 & $4.12(1.24)$ & $3.00(1.10)$ & $6.11(1.47)$ & 3.01 $(1.12)$ \\
\hline 2.5 & $3.24(0.88)$ & $\mathbf{2 . 3 7}(0.74)$ & $4.82(1.01)$ & $\mathbf{2 . 3 6}(0.74)$ \\
\hline \multirow[t]{2}{*}{3} & $2.69(0.66)$ & $\mathbf{1 . 9 9}(0.58)$ & $4.04(0.78)$ & $\mathbf{2 . 0 0}(0.58)$ \\
\hline & \multicolumn{2}{|c|}{$p=10$} & \multicolumn{2}{|c|}{$p=20$} \\
\hline 0 & $198.63(174.77)$ & $\mathbf{1 9 9 . 3 3}(197.00)$ & $199.75(150.45)$ & $\mathbf{2 0 0 . 3 5}(195.73)$ \\
\hline 0.5 & $42.70(21.19)$ & $21.46(17.41)$ & $55.80(21.30)$ & $\mathbf{2 1 . 9 0}(18.42)$ \\
\hline 1 & $18.68(5.63)$ & $\mathbf{7 . 3 1}(4.27)$ & $27.27(6.40)$ & $\mathbf{7 . 3 8}(4.26)$ \\
\hline 1.5 & $11.92(2.77)$ & 4.24(1.89) & $17.98(3.27)$ & 4.22(1.82) \\
\hline 2 & $8.84(1.71)$ & 3.01(1.09) & $13.49(2.03)$ & 3.02(1.10) \\
\hline 2.5 & $7.02(1.20)$ & $\mathbf{2 . 3 8}(0.75)$ & $10.79(1.46)$ & $\mathbf{2 . 3 7}(0.74)$ \\
\hline \multirow[t]{2}{*}{3} & $5.88(0.92)$ & $\mathbf{1 . 9 9}(0.57)$ & $9.053(1.11)$ & $\mathbf{2 . 0 0}(0.58)$ \\
\hline & \multicolumn{2}{|c|}{$p=2$} & \multicolumn{2}{|c|}{$p=5$} \\
\hline 0 & $498.50(496.41)$ & 489.447(482.85) & $500.07(482.47)$ & 498.65(487.34) \\
\hline 0.5 & $39.47(29.86)$ & 30.62(25.29) & $46.97(29.35)$ & $\mathbf{3 0 . 9 9}(25.23)$ \\
\hline 1 & $11.94(5.51)$ & $\mathbf{9 . 1 5}(5.02)$ & $16.26(5.86)$ & $\mathbf{9 . 1 2}(5.05)$ \\
\hline 1.5 & $6.87(2.35)$ & $\mathbf{5 . 1 4}(2.10)$ & $9.83(2.70)$ & $\mathbf{5 . 1 3}(2.10)$ \\
\hline 2 & $4.84(1.37)$ & 3.59(1.20) & $7.06(1.60)$ & $\mathbf{3 . 5 7}(1.20)$ \\
\hline 2.5 & $3.77(0.94)$ & $\mathbf{2 . 8 1}(0.82)$ & $5.56(1.10)$ & $\mathbf{2 . 8 0}(0.82)$ \\
\hline \multirow[t]{2}{*}{3} & $3.13(0.71)$ & $\mathbf{2 . 3 4}(0.61)$ & $4.65(0.84)$ & $\mathbf{2 . 0 5}(0.60)$ \\
\hline & \multicolumn{2}{|c|}{$p=10$} & \multicolumn{2}{|c|}{$p=20$} \\
\hline 0 & $500.70(468.09)$ & 491.22(489.49) & $497.20(435.10)$ & 496.65(496.75) \\
\hline 0.5 & $56.23(28.15)$ & $\mathbf{3 0 . 6 3}(25.11)$ & $73.38(28.28)$ & $\mathbf{3 0 . 6 9}(25.08)$ \\
\hline 1 & $22.36(6.43)$ & $\mathbf{9 . 1 3}(4.93)$ & $32.65(7.39)$ & $\mathbf{9 . 2 1}(4.98)$ \\
\hline 1.5 & $13.94(3.03)$ & $\mathbf{5 . 1 4}(2.08)$ & $21.08(3.59)$ & $\mathbf{5 . 1 4}(2.12)$ \\
\hline 2 & $10.19(1.86)$ & $\mathbf{3 . 6 0}(1.23)$ & $15.63(2.26)$ & $\mathbf{3 . 5 8}(1.21)$ \\
\hline 2.5 & $8.07(1.30)$ & $\mathbf{2 . 8 2}(0.83)$ & $12.45(1.55)$ & $\mathbf{2 . 8 1}(0.82)$ \\
\hline 3 & $6.71(0.98)$ & $\mathbf{2 . 3 4}(0.60)$ & $10.36(1.18)$ & $\mathbf{2 . 3 4}(0.61)$ \\
\hline
\end{tabular}

When $A R L_{0}=200$, it clearly appears that the new CUSUM procedure dominates Crosier's benchmark CUSUM chart. Not only the in-control $A R L$ is closer to the nominate $A R L_{0}=200$, 
but also the off-target $A R L s$ are uniformly shorter. In addition, this happens for all the tested dimensions $p \in\{2,5,10,20\}$. As for the case of $A R L_{0}=500$, the conclusion are similar except the in-control $A R L_{0}$ seem slightly underestimated by the new MCUSUM chart.

Secondly, we have made an experiment to compare the new MCUSUM chart MCn to a recent MCUSUM chart proposed in Golosnoy et al. (2009). Indeed, these authors have proposed several modifications of the MC1 chart introduced in Pignatiello and Runger (1990). One of these charts, called logarithmic chart, has been the most recommended one. More precisely, for each time epoch $t \geq 1$, let $n_{t}$ be the most recent time epoch before $t$ where the CUSUM process hit the lower boundary 0 , i.e. $S_{n}=0$. The logarithmic chart is then defined by

$$
\mathrm{LOG}_{t}=\max \left\{\ln \left\|S_{t-n_{t}, t}\right\|^{2}-\gamma g\left(n_{t}, k\right), 0\right\},
$$

where $S_{t-n_{t}, t}$ is the CUSUM from time epochs $n_{t}+1, \ldots, t$ and $g\left(n_{t}, d\right)=E\left(\ln \left\|S_{t-n_{t}, t}\right\|^{2}\right)$ which can be evaluated analytically using a series expansion. The chart will alarm when $\mathrm{LOG}_{t}>H$ for some UCL $H$.

The most difficult part in using this chart is the determination of the parameters $k$ and $\gamma$. Golosnoy et al. (2009) proposed a detailed study on this question. However, the study is limited to a small dimension $p=3$ and no general approach has been given when the distance $d$ varies. The final recommendation from the authors is to use $k \approx 0.8$ and $\gamma \approx \frac{1}{2}$ for "all" values of the distance $d$.

We follow such recommended values of $k$ for various values of the distance $d$ (which will have better $A R L$ performance than the one that fixes $k$ for all $d$ ). We also let $\gamma=\frac{1}{2}$ when dimension $p=3$, and compare its $A R L$ s to those of the new chart $\mathrm{MCn}$. The results are reported in Table 5. Here, the direction of shifts of the mean has been given to: $\mu=\frac{d}{\sqrt{p}}\left(\sigma_{1} u_{1}+\sigma_{2} u_{2}+\ldots+\sigma_{p} u_{p}\right)$ so that the distance $\left\|\mu-\mu_{0}\right\|=d$.

In this situation, the chart based on log norm has a slightly better performance. However, (i) 
Table 5: Comparison of $A R L$ performances between the new chart (MCn) and a logarithmic chart with $p=3$.

\begin{tabular}{c|c|c|c}
$d$ & \multicolumn{2}{|c|}{ Logarithmic Chart } & New chart MCn \\
\hline 0 & \multicolumn{2}{|c|}{$199.56(538.79)$} & $\mathbf{1 9 9 . 5 4}(197.42)$ \\
0.5 & $18.45(13.81)$ & $k=0.6$ & $\mathbf{2 1 . 5 0}(17.36)$ \\
1 & $7.33(4.22)$ & $k=0.8$ & $\mathbf{7 . 4 3}(4.26)$ \\
1.5 & $4.24(3.27)$ & $k=1.0$ & $\mathbf{4 . 2 5}(1.88)$ \\
2 & $2.92(2.03)$ & $k=1.2$ & $\mathbf{2 . 9 9}(1.10)$ \\
2.5 & $2.20(1.46)$ & $k=1.2$ & $\mathbf{2 . 3 7}(0.75)$ \\
3 & $1.76(1.11)$ & $k=1.4$ & $\mathbf{2 . 0 0}(0.57)$ \\
\hline
\end{tabular}

This $A R L$ performance in Table 5 is based on the fact we already know the change $d$ and then find the most optimal $k$ for the corresponding $d$. But in reality we have no pre-knowledge about $d$ and we have to pre-select the global $k$ at the beginning of the procedure, which will increase the $A R L s$ value in the Table 5. So the $A R L$ performance for log norm chart will degenerate; (ii) The s.e. for log norm chart seems rather larger compared with new chart especially when procedure is in-control or $d$ is large, which seems to indicate that log norm procedure is slightly unstable; (iii) The paper Golosnoy et al. (2009) provides the $A R L$ performance of log norm chart for $p=3$ only. If the dimension $p$ becomes larger, the $A R L$ performance may remains unknown. But the new method holds the similar $A R L$ performance whatever the dimension $p$ is and it already has quite similar ARLs' value compared with the best-performed ARLs of log norm chart; (iv) The log norm chart procedure has three parameters to estimate while the new method only have one parameter to evaluate. To sum up, we recommend the new chart rather than the chart based on $\log$ norm.

\section{Discussions and conclusions}

We have introduced a new MCUSUM chart where the target mean shift is assumed to be a weighted sum of principal directions of the population covariance matrix. This chart compares favourably to the benchmark chart of Crosier (1988). Meanwhile, we have provided new infor- 
mation on his historical chart with updated values of UCL $H$ and the associated $A R L$ function. We have also done a Monte-Carlo experiment to assess the accuracy of the well known Siegmund's approximations of the $A R L$ function when the observations are normal.

Although the assigned direction $\mu_{1}-\mu_{0}=\sigma_{1} u_{1}+\sigma_{2} u_{2}+\ldots+\sigma_{p} u_{p}$ is a reasonable prediction, we still need to handle diverse cases with other directions. To better discuss this problem, we set

$$
\mu_{1}-\mu_{0}=\frac{d}{\sqrt{p}}\left(\lambda_{1} \sigma_{1} u_{1}+\lambda_{2} \sigma_{2} u_{2}+\ldots+\lambda_{p} \sigma_{p} u_{p}\right)
$$

where $\lambda_{i} \geqslant 0(i=1,2, \ldots, p)$ and $\sum_{i=1}^{p} \lambda_{i}^{2}=p$. Then it satisfies that $\left\|\mu_{1}-\mu_{0}\right\|=d$. The parameter $\left(\lambda_{i}\right)$ are used here make the direction of shift $\mu_{1}-\mu_{0}$ more arbitrary. As a result, the given uncertain (with different $\lambda_{i}$ value) direction covers all the possibilities. The corresponding unit distance can be given as

$$
\mu_{1}-\mu_{0}=\frac{1}{\sqrt{p}}\left(\lambda_{1} \sigma_{1} u_{1}+\lambda_{2} \sigma_{2} u_{2}+\ldots+\lambda_{p} \sigma_{p} u_{p}\right)
$$

which satisfies that $\left\|\mu_{1}-\mu_{0}\right\|=1$ where we can find the optimal formula for sequential ratio statistic (or optimal $k$ ) for specific direction. It can be calculated that

$$
s_{n}=\frac{1}{\sqrt{p}}\left(\frac{\lambda_{1}}{\sigma_{1}} c_{n}^{1}+\frac{\lambda_{2}}{\sigma_{2}} c_{n}^{2}+\ldots \frac{\lambda_{p}}{\sigma_{p}} c_{n}^{p}\right)-\frac{1}{2} \quad\left(k=\frac{1}{2}\right)
$$

where $c_{n}^{j}=u_{j}^{T}\left(x_{n}-\mu_{0}\right)(j=1,2,3 \ldots p)$.

In addition, the $A R L$ can be also calculated as follows

$$
\mu^{\prime}=E\left(s_{n}\right)=\frac{1}{\sqrt{p}} \sum_{j=1}^{p} \frac{\lambda_{i} u_{j}^{T}}{\sigma_{j}}\left(\mu-\mu_{0}\right)-\frac{1}{2}=d-\frac{1}{2},
$$

and $\operatorname{VAR}\left(s_{n}\right)=1$. Fortunately enough, these values are the same as in the previous case with 
assigned direction $\left(\mu_{1}-\mu_{0}=\sigma_{1} u_{1}+\sigma_{2} u_{2}+\ldots+\sigma_{p} u_{p}\right)$, see Appendix for more details. So the final UCL $H$ and $A R L$ formula will also be the same as given in (11)-(12).

In general, we say that $A R L$ s' performance remains the same no matter what direction it changes with corresponding statistic $s_{n}$.

In conclusion, if accurate information on the direction of shift is known, we can accomodate it with the corresponding $\left(\lambda_{i}\right)$ and the formula of $S_{n}$ given above. Otherwise, we recommend the new chart MCn introduced in (9).

\section{Acknowledgments}

Jiaqi Chen's research is supported by National Natural Science Foundation of China, No. 11501147.

\section{References}

M. Basseville, I.V. Nikiforov, et al. Detection of Abrupt Changes: Theory and Application, volume 15. Prentice Hall Englewood Cliffs, 1993.

S. Bersimis, S. Psarakis, and J. Panaretos. Multivariate statistical process control charts: an overview. Quality and Reliability Engineering International, 23(5):517-543, 2007.

R.B. Crosier. Multivariate generalizations of cumulative sum quality-control schemes. Technometrics, pages 291-303, 1988.

V. Golosnoy, S. Ragulin, and W. Schmid. Multivariate CUSUM chart: properties and enhancements. AStA Advances in Statistical Analysis, 93(3):263-279, 2009.

J.D. Healy. A note on multivariate CUSUM procedures. Technometrics, pages 409-412, 1987.

E.S. Page. Continuous inspection schemes. Biometrika, 41(1/2):100-115, 1954. 
J.J. Pignatiello and G.C. Runger. Comparisons of multivariate CUSUM charts. Journal of Quality Technology, 22(3):173-186, 1990.

G.C. Runger and M.C. Testik. Multivariate extensions to cumulative sum control charts. Quality and Reliability Engineering International, 20(6):587-606, 2004.

D. Siegmund. Sequential Analysis: Tests and Confidence Intervals. Springer, 1985.

A. Wald. Sequential Analysis. Dover Pubns, 2004.

W.H. Woodall and M.M. Ncube. Multivariate CUSUM quality-control procedures. Technometrics, pages 285-292, 1985.

\section{A Derivation of (14)-(15)}

Since $\mathbf{x}_{\mathbf{n}} \sim N(\mu, \Sigma)$, we have

$$
c_{n}^{j} \sim N\left(u_{j}^{T}\left(\mu-\mu_{0}\right), u_{j}^{T} \Sigma u_{j}\right)=N\left(u_{j}^{T}\left(\mu-\mu_{0}\right), \sigma_{j}^{2}\right), \quad \text { and } \quad \frac{1}{\sigma_{j}} c_{n}^{j} \sim N\left(\frac{u_{j}^{T}}{\sigma_{j}}\left(\mu-\mu_{0}\right), 1\right) .
$$

Therefore, $s_{n} \sim N\left(\frac{1}{\sqrt{p}} \sum_{j=1}^{p} \frac{u_{j}^{T}}{\sigma_{j}}\left(\mu-\mu_{0}\right)-\frac{1}{2}, 1\right)$, and $\sigma^{\prime 2}=\operatorname{VAR}\left(s_{n}\right)=1$, where $\left\{\sigma_{i}^{2}\right\}_{1 \leq i \leq p}$ are the eigenvalues of the covariance matrix $\Sigma$ while $u_{i}(i=1,2,3 \ldots p)$ are the eigenvectors of $\Sigma$.

The direction of the mean shift is distributed as a weighted sum of principle component directions, which can be written as

$$
\mu-\mu_{0}=k\left(\sigma_{1} u_{1}+\sigma_{2} u_{2}+\ldots+\sigma_{p} u_{p}\right) .
$$

Since $d^{2}=\left(\mu_{1}-\mu_{0}\right)^{T} \Sigma^{-1}\left(\mu_{1}-\mu_{0}\right)=k^{2} p$ (where the relationship between $d$ and $p$ can be 
seen), we have $\mu-\mu_{0}=\frac{d}{\sqrt{p}}\left(\sigma_{1} u_{1}+\sigma_{2} u_{2}+\ldots+\sigma_{p} u_{p}\right)$. Therefore,

$$
\mu^{\prime}=\mathrm{E}\left(s_{n}\right)=\frac{1}{\sqrt{p}} \sum_{j=1}^{p} \frac{u_{j}^{T}}{\sigma_{j}}\left(\mu-\mu_{0}\right)=\frac{d}{\sqrt{p}} \frac{1}{\sqrt{p}} \sum_{j=1}^{p} \frac{u_{j}^{T}}{\sigma_{j}} \sigma_{j} u_{j}-\frac{1}{2}=d-\frac{1}{2}
$$

By substituting these values of $\mu^{\prime}$ and $\sigma^{\prime 2}$ in Sigemund's formula (11)-(12), we obtain the $A R L$ formula in (14)-(15).

\section{B Empirical assessment of the accuracy of Siegmund's $A R L$ approximations (14)-(15)}

We have made simulations to examine Siegmund's approximation by mainly consider four different covariance matrices. Each matrix has its own eigenvectors and thus eigenvector space. For each group, we draw samples with a size of 10000 and simulate the CUSUM procedure until the sum of the sample exceeds the control limit.

- Case 1: Identity covariance matrix

Each sample $\mathbf{x}_{n}$ is generated from $N\left(\mu, I_{p}\right)$ with on-target mean $\mu_{0}$ and covariance matrix $I_{p}$. Then we can get eigenvalues $\sigma_{1}^{2}=\sigma_{2}^{2}=\ldots=\sigma_{p}^{2}=1$ as well as eigenvectors $u_{1}=$ $(1,0,0, \ldots, 0)^{T} u_{2}=(0,1,0, \ldots, 0)^{T} \ldots . . u_{p}=(0,0,0, \ldots, 1)^{T}$.Then the change direction for mean vector has the form $\mu-\mu_{0}=\left(\frac{d}{\sqrt{p}}, \frac{d}{\sqrt{p}}, \frac{d}{\sqrt{p}}, \ldots, \frac{d}{\sqrt{p}}\right)^{T}$ and we accumulate the $s_{n}=\frac{1}{\sqrt{p}} \sum_{i=1}^{p}\left(\mathbf{x}_{n}-\mu_{\mathbf{0}}\right)_{[i]}-\frac{1}{2}$ where $(\cdot)_{[i]}$ denotes the $i$ th element of the vector $(\cdot)$.

- Case 2: Diagonal matrix with unequal elements

Each sample $\mathbf{x}_{\mathbf{n}}$ is generated from $N(\mu, A)$ with on-target mean $\mu_{0}$ and covariance matrix 
$A$ where the sum of eigenvalues equals to $p$ :

$$
A=\left(\begin{array}{ccccc}
\frac{2}{1+p} & 0 & \cdots & 0 & 0 \\
0 & 2 \times \frac{2}{1+p} & \vdots & \vdots & \vdots \\
0 & 0 & \ddots & 0 & 0 \\
\vdots & \vdots & \vdots & (p-1) \times \frac{2}{1+p} & 0 \\
0 & 0 & \cdots & 0 & p \times \frac{2}{1+p}
\end{array}\right)
$$

Then we can get eigenvalues $\sigma_{1}=\sqrt{\frac{2}{1+p}} \sigma_{2}=\sqrt{\frac{4}{1+p}} \ldots \sigma_{i}=\sqrt{\frac{2 i}{1+p}} \ldots \sigma_{p}=\sqrt{\frac{2 p}{1+p}}$ as well as eigenvectors $u_{1}=(1,0,0, \ldots, 0)^{T} u_{2}=(0,1,0, \ldots, 0)^{T} \ldots . . u_{p}=(0,0,0, \ldots, 1)^{T}$. The eigenvalues gradually increase along with the dimensions. Then the change direction for mean vector has the form $\mu-\mu_{0}=\frac{d}{\sqrt{p}}\left(\sqrt{\frac{2}{1+p}}, \ldots, \sqrt{\frac{2 i}{1+p}}, \ldots, \sqrt{\frac{2 p}{1+p}}\right)^{T}$ and we accumulate the $s_{n}=\sqrt{\frac{1+p}{2 p}} \sum_{i=1}^{p} \frac{1}{\sqrt{i}}\left(\mathbf{x}_{n}-\mu_{\mathbf{0}}\right)-\frac{1}{2}$.

- Case 3 and 4: Toeplitz-type matrices (AR(1) auto-correlation matrices)

Each sample $x_{n}$ is generated from $N(\mu, A)$ with on-target mean $\mu_{0}$ and covariance matrix $A$ where

$$
A=\left(\begin{array}{ccccc}
1 & \rho & \rho^{2} & \cdots & \rho^{p-1} \\
\rho & 1 & \rho & \cdots & \rho^{p-2} \\
\rho^{2} & \rho & 1 & \cdots & \rho^{p-3} \\
\vdots & \vdots & \vdots & \ddots & \vdots \\
\rho^{p-1} & \rho^{p-2} & \rho^{p-3} & \cdots & 1
\end{array}\right)
$$

Here the eigenvectors are no more the canonical ones. Two values of $\rho$ have been tested:

Case 3: $\rho=0.75$,

Case 4: $\rho=0.2$. 
We get the eigenvalues and eigenvectors via Matlab. Then accumulate the sum as procedure described above.

We finally draw 10000 samples and get 10000 run lengths for each group. The average run length of 10000 samples for each group as well as standard deviation are reported in Table 6 and Table 7.

It can be seen that empirical $A R L$ s for four groups under each dimension are nearly identical. Also, the ARLs for all dimensions under each group are almost the same. When compared with Siegmund's approximation, $A R L$ values given by Siegmund's approximation seem slightly smaller than the empirical ARLs with however a difference limited to $2 \%$ at most. This proves that the Siegmund's approximation is accurate enough for use in practice. 
Table 6: $A R L$ comparison among 4 cases when $A R L_{0}=200$.

\begin{tabular}{|c|c|c|c|c|c|c|c|c|c|c|}
\hline \multirow{9}{*}{$p=2$} & \multirow{3}{*}{ Case 1} & $d$ & 0 & 0.5 & 1 & 1.5 & 2 & 2.5 & 3 & 3.5 \\
\hline & & $A R L$ & 198.07 & 21.67 & 7.31 & 4.27 & 3.00 & 2.37 & 1.99 & 1.73 \\
\hline & & s.d. & 196.10 & 17.65 & 4.18 & 1.90 & 1.10 & 0.74 & 0.58 & 0.52 \\
\hline & \multirow[t]{2}{*}{ Case 2} & $A R L$ & 198.20 & 22.02 & 7.34 & 4.26 & 3.01 & 2.37 & 1.99 & 1.73 \\
\hline & & s.d. & 193.87 & 18.08 & 4.21 & 1.86 & 1.10 & 0.75 & 0.58 & 0.53 \\
\hline & \multirow[t]{2}{*}{ Case 3} & $A R L$ & 195.75 & 21.87 & 7.40 & 4.23 & 3.02 & 2.38 & 1.99 & 1.73 \\
\hline & & s.d. & 192.01 & 17.81 & 4.43 & 1.87 & 1.10 & 0.74 & 0.58 & 0.52 \\
\hline & \multirow[t]{2}{*}{ Case 4} & $A R L$ & 199.55 & 21.63 & 7.41 & 4.26 & 3.00 & 2.38 & 2.00 & 1.73 \\
\hline & & s.d. & 194.31 & 17.81 & 4.29 & 1.88 & 1.08 & 0.75 & 0.57 & 0.53 \\
\hline \multirow{9}{*}{$p=5$} & \multirow{3}{*}{ Case 1} & $d$ & 0 & 0.5 & 1 & 1.5 & 2 & 2.5 & 3 & 3.5 \\
\hline & & $A R L$ & 199.14 & 21.73 & 7.33 & 4.24 & 3.01 & 2.36 & 2.00 & 1.74 \\
\hline & & s.d. & 196.84 & 17.46 & 4.24 & 1.87 & 1.12 & 0.74 & 0.58 & 0.53 \\
\hline & \multirow[t]{2}{*}{ Case 2} & $A R L$ & 198.76 & 21.69 & 7.37 & 4.26 & 3.00 & 2.37 & 1.99 & 1.74 \\
\hline & & s.d. & 196.90 & 17.82 & 4.31 & 1.86 & 1.09 & 0.75 & 0.57 & 0.51 \\
\hline & \multirow[t]{2}{*}{ Case 3} & $A R L$ & 201.27 & 21.79 & 7.36 & 4.23 & 3.01 & 2.37 & 1.99 & 1.73 \\
\hline & & s.d. & 198.86 & 17.93 & 4.21 & 1.85 & 1.12 & 0.74 & 0.57 & 0.52 \\
\hline & \multirow[t]{2}{*}{ Case 4} & $A R L$ & 199.27 & 21.70 & 7.37 & 4.24 & 3.01 & 2.39 & 1.99 & 1.73 \\
\hline & & s.d. & 196.30 & 17.50 & 4.28 & 1.86 & 1.09 & 0.76 & 0.58 & 0.53 \\
\hline \multirow{9}{*}{$p=10$} & \multirow{3}{*}{ Case 1} & $d$ & 0 & 0.5 & 1 & 1.5 & 2 & 2.5 & 3 & 3.5 \\
\hline & & $A R L$ & 199.33 & 21.46 & 7.31 & 4.24 & 3.01 & 2.38 & 1.99 & 1.73 \\
\hline & & s.d. & 197.00 & 17.41 & 4.27 & 1.89 & 1.09 & 0.75 & 0.57 & 0.51 \\
\hline & \multirow[t]{2}{*}{ Case 2} & $A R L$ & 190.94 & 21.57 & 7.46 & 4.25 & 3.00 & 2.36 & 2.00 & 1.73 \\
\hline & & s.d. & 197.92 & 17.55 & 4.39 & 1.89 & 1.07 & 0.74 & 0.573 & 0.53 \\
\hline & \multirow[t]{2}{*}{ Case 3} & $A R L$ & 198.96 & 21.67 & 7.36 & 4.25 & 3.02 & 2.37 & 2.00 & 1.72 \\
\hline & & s.d. & 192.67 & 17.88 & 4.29 & 1.83 & 1.08 & 0.74 & 0.58 & 0.52 \\
\hline & \multirow[t]{2}{*}{ Case 4} & $A R L$ & 201.66 & 21.75 & 7.37 & 4.23 & 3.01 & 2.36 & 1.99 & 1.72 \\
\hline & & s.d. & 199.50 & 17.73 & 4.22 & 1.86 & 1.09 & 0.74 & 0.58 & 0.53 \\
\hline \multirow{9}{*}{$p=20$} & \multirow{3}{*}{ Case 1} & $d$ & 0 & 0.5 & 1 & 1.5 & 2 & 2.5 & 3 & 3.5 \\
\hline & & $A R L$ & 200.35 & 21.90 & 7.38 & 4.22 & 3.02 & 2.37 & 2.00 & 1.73 \\
\hline & & s.d. & 195.73 & 18.42 & 4.26 & 1.82 & 1.10 & 0.74 & 0.58 & 0.53 \\
\hline & \multirow[t]{2}{*}{ Case 2} & $A R L$ & 198.91 & 21.81 & 7.34 & 4.24 & 3.01 & 2.37 & 1.99 & 1.73 \\
\hline & & s.d. & 198.24 & 17.59 & 4.25 & 1.86 & 1.08 & 0.75 & 0.57 & 0.52 \\
\hline & \multirow[t]{2}{*}{ Case 3} & $A R L$ & 196.53 & 21.55 & 7.35 & 4.23 & 3.00 & 2.37 & 1.99 & 1.72 \\
\hline & & s.d. & 192.63 & 17.84 & 4.22 & 1.85 & 1.09 & 0.75 & 0.59 & 0.53 \\
\hline & \multirow[t]{2}{*}{ Case 4} & $A R L$ & 199.09 & 21.62 & 7.46 & 4.25 & 3.00 & 2.37 & 2.00 & 1.73 \\
\hline & & s.d. & 194.79 & 17.71 & 4.39 & 1.89 & 1.09 & 0.74 & 0.57 & 0.53 \\
\hline \multirow{2}{*}{\multicolumn{3}{|c|}{ Siegmund's approximation }} & 0 & 0.5 & 1 & 1.5 & 2 & 2.5 & 3 & 3.5 \\
\hline & & & 200.01 & 21.72 & 7.34 & 4.16 & 2.88 & 2.21 & 1.78 & 1.50 \\
\hline
\end{tabular}


Table 7: $A R L$ comparison among 4 cases when $A R L_{0}=500$.

\begin{tabular}{|c|c|c|c|c|c|c|c|c|c|c|}
\hline \multirow{9}{*}{$p=2$} & \multirow{3}{*}{ Case 1} & $d$ & 0 & 0.5 & 1 & 1.5 & 2 & 2.5 & 3 & 3.5 \\
\hline & & $\overline{A R L}$ & 489.45 & 30.62 & 9.15 & 5.13 & 3.59 & 2.81 & 2.34 & 2.05 \\
\hline & & s.d. & 482.85 & 25.29 & 5.02 & 2.10 & 1.20 & 0.82 & 0.61 & 0.47 \\
\hline & \multirow[t]{2}{*}{ Case 2} & $A R L$ & 501.68 & 30.80 & 9.17 & 5.10 & 3.59 & 2.80 & 2.33 & 2.04 \\
\hline & & s.d. & 494.92 & 25.39 & 5.00 & 2.07 & 1.23 & 0.83 & 0.61 & 0.47 \\
\hline & \multirow[t]{2}{*}{ Case 3} & $A R L$ & 489.22 & 31.01 & 9.17 & 5.12 & 3.59 & 2.81 & 2.34 & 2.05 \\
\hline & & s.d. & 480.94 & 24.72 & 5.03 & 2.12 & 1.23 & 0.83 & 0.60 & 0.46 \\
\hline & \multirow[t]{2}{*}{ Case 4} & $A R L$ & 497.78 & 31.06 & 9.15 & 5.15 & 3.58 & 2.81 & 2.33 & 2.05 \\
\hline & & s.d. & 497.98 & 25.55 & 4.96 & 2.11 & 1.19 & 0.82 & 0.59 & 0.47 \\
\hline \multirow{9}{*}{$p=5$} & \multirow{3}{*}{ Case 1} & $d$ & 0 & 0.5 & 1 & 1.5 & 2 & 2.5 & 3 & 3.5 \\
\hline & & $A R L$ & 498.65 & 30.99 & 9.12 & 5.13 & 3.57 & 2.80 & 2.33 & 2.05 \\
\hline & & s.d. & 487.34 & 25.23 & 5.05 & 2.10 & 1.20 & 0.82 & 0.60 & 0.46 \\
\hline & \multirow[t]{2}{*}{ Case 2} & $A R L$ & 496.07 & 30.50 & 9.10 & 5.11 & 3.61 & 2.80 & 2.34 & 2.06 \\
\hline & & s.d. & 496.07 & 24.93 & 4.94 & 2.07 & 1.23 & 0.82 & 0.60 & 0.47 \\
\hline & \multirow[t]{2}{*}{ Case 3} & $A R L$ & 491.42 & 30.94 & 9.17 & 5.10 & 3.58 & 2.80 & 2.34 & 2.05 \\
\hline & & s.d. & 489.10 & 25.59 & 5.04 & 2.08 & 1.21 & 0.83 & 0.61 & 0.47 \\
\hline & \multirow[t]{2}{*}{ Case 4} & $A R L$ & 498.99 & 30.69 & 9.14 & 5.10 & 3.58 & 2.80 & 2.34 & 2.05 \\
\hline & & s.d. & 484.47 & 25.19 & 4.98 & 2.06 & 1.20 & 0.82 & 0.60 & 0.46 \\
\hline \multirow{9}{*}{$p=10$} & \multirow{3}{*}{ Case 1} & $d$ & 0 & 0.5 & 1 & 1.5 & 2 & 2.5 & 3 & 3.5 \\
\hline & & $A R L$ & 491.22 & 30.63 & 9.13 & 5.14 & 3.60 & 2.82 & 2.34 & 2.05 \\
\hline & & s.d. & 489.49 & 25.11 & 4.93 & 2.08 & 1.23 & 0.83 & 0.60 & 0.47 \\
\hline & \multirow[t]{2}{*}{ Case 2} & $A R L$ & 495.82 & 30.65 & 9.06 & 5.14 & 3.60 & 2.80 & 2.35 & 2.04 \\
\hline & & s.d. & 487.09 & 25.12 & 4.97 & 2.12 & 1.20 & 0.83 & 0.60 & 0.47 \\
\hline & \multirow[t]{2}{*}{ Case 3} & $A R L$ & 488.45 & 30.65 & 9.09 & 5.09 & 3.58 & 2.81 & 2.34 & 2.04 \\
\hline & & s.d. & 481.58 & 25.28 & 4.96 & 2.11 & 1.21 & 0.82 & 0.61 & 0.47 \\
\hline & \multirow[t]{2}{*}{ Case 4} & $A R L$ & 494.20 & 30.60 & 9.06 & 5.14 & 3.61 & 2.81 & 2.33 & 2.04 \\
\hline & & s.d. & 484.87 & 25.18 & 4.99 & 2.14 & 1.22 & 0.82 & 0.60 & 0.46 \\
\hline \multirow{9}{*}{$p=20$} & \multirow{3}{*}{ Case 1} & $d$ & 0 & 0.5 & 1 & 1.5 & 2 & 2.5 & 3 & 3.5 \\
\hline & & $A R L$ & 496.65 & 30.69 & 9.21 & 5.14 & 3.58 & 2.81 & 2.34 & 2.04 \\
\hline & & s.d. & 496.75 & 25.08 & 4.98 & 2.12 & 1.21 & 0.82 & 0.61 & 0.46 \\
\hline & \multirow[t]{2}{*}{ Case 2} & $A R L$ & 501.42 & 30.34 & 9.14 & 5.11 & 3.59 & 2.80 & 2.34 & 2.05 \\
\hline & & s.d. & 492.40 & 24.53 & 5.13 & 2.11 & 1.22 & 0.82 & 0.61 & 0.47 \\
\hline & \multirow[t]{2}{*}{ Case 3} & $A R L$ & 491.51 & 30.69 & 9.10 & 5.15 & 3.58 & 2.79 & 2.34 & 2.05 \\
\hline & & s.d. & 495.18 & 25.02 & 4.93 & 2.13 & 1.23 & 0.81 & 0.61 & 0.47 \\
\hline & \multirow[t]{2}{*}{ Case 4} & $A R L$ & 502.65 & 30.91 & 9.15 & 5.11 & 3.57 & 2.81 & 2.33 & 2.05 \\
\hline & & s.d. & 505.59 & 25.42 & 5.11 & 2.08 & 1.20 & 0.83 & 0.60 & 0.47 \\
\hline \multirow{2}{*}{\multicolumn{3}{|c|}{ Siegmund's approximation }} & 0 & 0.5 & 1 & 1.5 & 2 & 2.5 & 3 & 3.5 \\
\hline & & & 498.96 & 30.75 & 9.10 & 5.05 & 3.47 & 2.65 & 2.14 & 1.79 \\
\hline
\end{tabular}

